To the Editors:

\title{
A case series of three Sri Lankan families with hereditary breast and ovarian cancer syndrome due to pathogenic germline mutations in the $B R C A 1$ gene
}

\author{
N D Sirisena ${ }^{1}$, B Gautam ${ }^{1}$, N D W Dissanayake ${ }^{1}$, V H W Dissanayake ${ }^{1,2}$ \\ Ceylon Medical Journal 2017; 62: 65-66 \\ DOI: http://doi.org/10.4038/cmj.v62i1.8437
}

Several risk factors predispose to hereditary breast cancer, the most potent being mutations in the breast cancer susceptibility genes, BRCA 1 and BRCA 2 (BRCA1/2). A germ line mutation in either of these genes confers a high life time risk of developing breast cancer in mutation carriers; $46-87 \%$ for BRCA1 and $26-84 \%$ for $B R C A 2$ up to the age of 70 years [2].

We analysed retrospectively collected clinicopathological data and genetic mutations detected in families with hereditary breast cancer, referred to a diagnostic centre of a fee levying hospital undertaking BRCA gene testing from January 2010 to December 2015. The data is from an anonymized database. Approval was obtained from the Ethics Review Committee of the Faculty of Medicine, University of Colombo, Sri Lanka. After providing pre-test counselling and obtaining written informed consent, patients with breast cancer were tested for BRCA1 gene mutations by direct DNA sequencing.

In three families with hereditary breast cancer, pathogenic germline mutations in the BRCA1 gene were identified. All affected patients had invasive duct carcinoma of the breast. Family 1 had breast cancer due to a deleterious frame shift mutation resulting in premature stop codon in exon 21 (c.5289delG; p.Leu1764Terfs) while family 2 had breast cancer due to a nonsense mutation in exon 11 (c.2719G>T; p.Glu907Ter). Family 3 had breast and ovarian cancer due to a deleterious frame shift mutation resulting in premature stop codon in exon 2 (c.68-69delAG; p.Glu23ValfsX17). All three pathogenic germline $B R C A 1$ gene mutations have been previously reported in families with hereditary breast cancer [2, 4].
The BRCA1 mutation identified in family 3 (с.6869delAG; p.Glu23ValfsX17) is the most frequently reported founder mutation among Ashkenazi Jewish descendants [4]. The first large-scale study to report mutations in the BRCA1 gene in the Sri Lankan population was conducted by De Silva et al. [2]. They identified 19 sequence variants in the BRCA1 gene and two novel deleterious frame-shift mutations, namely, c.3086delT in exon 11 and c.5289delG in exon 21 as well as one possibly pathogenic novel missense mutation, c.856T $>\mathrm{G}$ in exon 11. In a related Sri Lankan study, mutations and sequence variants in the BRCA2 gene were studied [3]. Two novel pathogenic frame-shift additions resulting in a premature stop codon (c.2403 insA/exon 11, c.2667 insT/exon 11) and possibly pathogenic two novel missense mutations (c.1191 A>C/exon 10, c.5695 A>C/ exon 11) were identified. Further studies need to be conducted to identify other pathogenic BRCA1/2 gene mutations in a larger cohort of Sri Lankan patients, and to characterise the genotype-phenotype spectrum of hereditary breast cancer. Such knowledge will be vital not only for the treatment and follow up of patients but more importantly for screening of individuals with a hereditary predisposition to cancer. Characterization of such germline mutations will allow at risk asymptomatic family members to make informed decisions regarding reproduction, lifestyle and adoption of preventive, riskreduction measures leading to improved survival and quality of life.

\section{Conflicts of interest}

There are no conflicts of interest.

${ }^{1}$ Human Genetics Unit, Faculty of Medicine, University of Colombo and ${ }^{2}$ Asiri Centre for Genomic and Regenerative Medicine, Colombo, Sri Lanka.

Correspondence: VHWD, e-mail: <vajira@anat.cmb.ac.lk>. Received 3 May 2016 and revised version accepted 14 December 2016.

This is an open-access article distributed under the terms of the Creative Commons Attribution License, which permits unrestricted use, distribution, and reproduction in any medium, provided the original author and source are credited. 


\section{References}

1. National Cancer Control Programme. Cancer Incidence Data Sri Lanka 2009, 11th Publication. Colombo: National Cancer Control Programme 2015; 10.

2. De Silva W, Tennekoon KH, Karunanayake EH, et al. Novel sequence variants and a high frequency of recurrent polymorphisms in BRCA1 gene in Sri Lankan breast cancer patients and at risk individuals. BMC Cancer 2008; 8: 1-8.

3. Kim H, Choi DH. Distribution of BRCA1 and BRCA2 Mutations in Asian Patients with Breast Cancer. Journal of Breast Cancer 2013; 16: 357-65.

4. Kwong A, Shin VY, Ho JCW, et al. Comprehensive spectrum of BRCA1 and BRCA2 deleterious mutations in breast cancer in Asian countries. J Med Genet 2016; 53: 15-23.

5. De Silva S, Tennekoon KH, Karunanayake EH, De Silva W, Amarasinghe I, Angunawala P. Novel sequence variants and common recurrent polymorphisms of $B R C A 2$ in Sri Lankan breast cancer patients and a family with BRCA1 mutations. Exp Ther Med 2011; 2: 1163-70.

6. De Silva S, Tennekoon KH, Karunanayake EH, Amarasinghe I, Angunawala P. Analysis of BRCA1 and BRCA2 large genomic rearrangements in Sri Lankan familial breast cancer patients and at risk individuals. BMC Res Notes 2014; 7: 344. 